\title{
Transmit Waveform Optimization for Spatial-Frequency Diversity MIMO Radar in the Presence of Clutter
}

\author{
Yonghao Tang, Xiaofeng Ma, Weixing Sheng, and Yubing Han \\ School of Electronic and Optical Engineering, Nanjing University of Science and Technology, Nanjing 210094, China \\ Correspondence should be addressed to Xiaofeng Ma; maxiaofeng@njust.edu.cn
}

Received 25 February 2014; Revised 1 June 2014; Accepted 16 June 2014; Published 1 July 2014

Academic Editor: Mathini Sellathurai

Copyright (C) 2014 Yonghao Tang et al. This is an open access article distributed under the Creative Commons Attribution License, which permits unrestricted use, distribution, and reproduction in any medium, provided the original work is properly cited.

\begin{abstract}
Benefitting from the independent target echoes of diversity channels, diversity MIMO radar can efficiently improve system performance, such as target detection and parameter estimation. Due to the fact that the RCS (radar cross section) of complex target may vary with the different transmitted carrier frequencies and array geometries, many recent researches study at the background of diversity MIMO radar equipped with widely separated array antennas or working at multiple carrier frequencies, respectively. In this paper, a new MIMO radar system combining the spatial and frequency diversities is investigated in the presence of signaldependent clutter, which is called spatial-frequency diversity MIMO radar. With the prior information of target and clutter, a new method for joint optimization of transmitted waveforms and receiving filters is proposed to enhance the target detection ability of spatial-frequency diversity MIMO radar. Inspired by the MIMO communication system, the water-filling algorithm is introduced into the transmitted energy allocation problem for each carrier frequency channel. Simulation results show that the proposed system has a better performance in output signal-to-clutter-noise ratio (SCNR) compared to conventional diversity MIMO radar system.
\end{abstract}

\section{Introduction}

Recently, the transmitted waveforms optimization problem of MIMO radar is a very popular issue in radar research field [1-5]. In conventional MIMO radar, the target diversity information of independent channels can be extracted by matched filters. The extra degrees of freedom brought by diversity information enable the system to obtain better performance in target detection and parameter estimation [6-8]. However, in the presence of clutter, the conventional MIMO radar is unable to suppress clutter effectively for the reason of the intrinsic correlation between the clutter and target echoes. Consequently, the joint optimization of transmitted waveforms and receiving filters for MIMO radar to suppress the clutter has attracted the attention of more and more researchers [9-11].

Because of its ability to transmit arbitrary waveforms, MIMO radar can obtain plenty of diversity information of target echoes. It is possible to suppress the clutters effectively by properly utilizing the diversity information. According to the types of diversity information, diversity MIMO radar can be divided into two types: spatial diversity MIMO radar
$[12,13]$ and frequency diversity MIMO radar [14, 15]. For spatial diversity MIMO radar, in order to observe target from different directions, the array antennas are often widely distributed. According to $[7,16]$, MIMO radar system will obtain the different RCS of target from different directions when the spacing between every two antennas satisfies

$$
d_{t} \geq \frac{\lambda D}{d}
$$

where $d_{t}$ denotes the spacing between two antennas, $\lambda$ is the wavelength of carrier frequency, $D$ is the target distance departed to array, and $d$ represents target size. For frequency diversity MIMO radar, the system obtains the independent target echoes by transmitting probing signals of different carrier frequencies. Even though the array antennas may be co-located distributed, the frequency diversity can be obtained only if the working frequency difference of two frequency channels satisfies $[16,17]$

$$
\Delta f \geq \frac{c}{2 d},
$$

where $\Delta f$ denotes the carrier frequency difference of two frequency channels and $c$ represents the speed of light. 

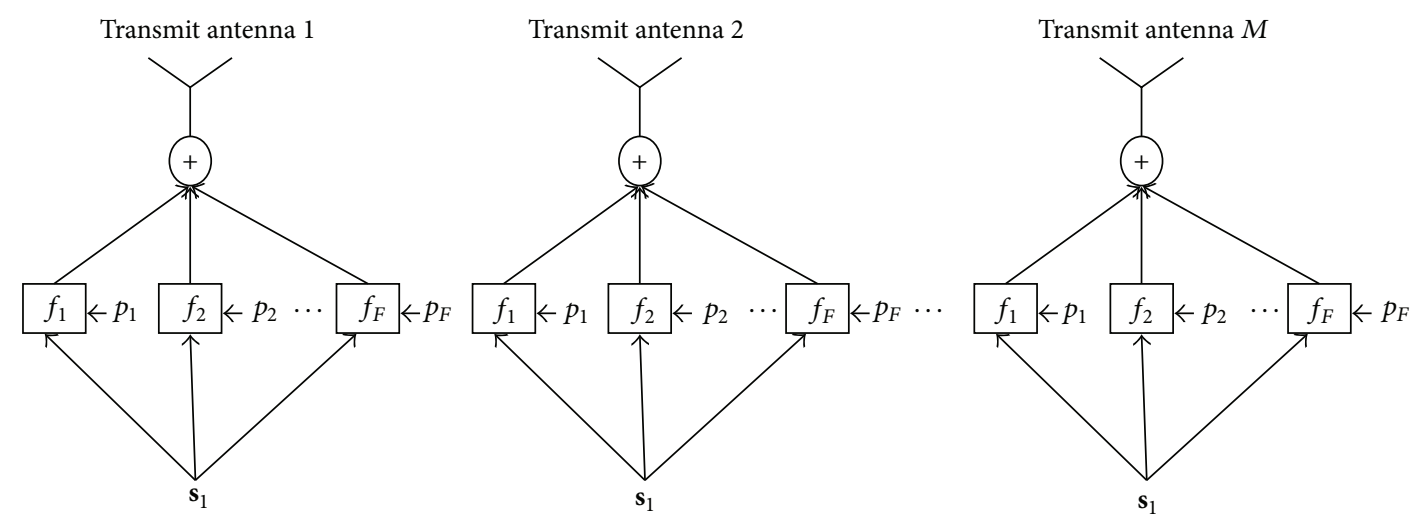

FIgURE 1: The structure of transmit array.

In this paper, spatial-frequency diversity MIMO radar system is investigated, which combines the advantages brought by spatial and frequency diversities. The structure of spatial-frequency diversity MIMO radar is shown in Figures 1 and 2 . The array antennas are widely distributed. The spacing between every two antennas satisfies (1), so that the spatial diversity information can be obtained. Meanwhile, each antenna transmits probing signals of $F$ carrier frequencies. The frequency difference of every two frequency channels satisfies (2) to obtain the frequency diversity information. So much diversity information provides much more extra degrees of freedom to design transmitted waveforms, receiving filters and energy allocation of frequency channels, which makes it possible for spatial-frequency diversity MIMO radar to achieve better system performance than conventional diversity MIMO radar. However, the difficulty is that the joint optimization of transmitted waveforms and receiving filters is not a convex optimization problem, which cannot get the closed-form solution easily. In addition, the energy allocation optimization of different frequency channels is a new problem that is faced by spatial-frequency diversity MIMO radar.

So far, the transmitted waveforms optimization techniques are mostly developed for MIMO radar with widely distributed antennas, which only take advantage of the spatial diversity information $[1,3,4,12,13,18]$. In order to improve the performance of parameter estimation, the MMSE, NMSE, and MI methods are investigated for spatial diversity MIMO radar in [1] and the references therein. The methods proposed in [18] are also developed for spatial diversity MIMO radar to improve the target detection performance. Unfortunately, all these methods are not extended to spatial-frequency diversity MIMO radar yet. Unlike these references, this paper proposes a joint optimization method of transmitted waveforms and receiving filters for spatial-frequency diversity MIMO radar to improve the target detection performance against clutters. A similar iteration algorithm designed for traditional radar system is proposed in [19]. However, it cannot be directly applied in MIMO radar especially the proposed spatialfrequency diversity MIMO radar. In the proposed method, the rule of maximum output SCNR is applied to optimize the transmitted waveforms and receiving filters jointly. Under the premise that the target and clutter are known as priors, an iterative algorithm is proposed to maximize the output SCNR of system by optimizing the transmitted waveforms and receiving filters alternatively. The output SCNR in each step of the iterative algorithm is nondecreasing, so that the convergence of the proposed method is guaranteed. Meanwhile, inspired by the MIMO communication theory, the water-filling algorithm is introduced to the optimization problem of energy allocation for different frequency channels. Simulation results show that the water-filling algorithm significantly improves the system performance. Combining these methods, spatial-frequency diversity MIMO radar achieves a greatly improved target detection performance compared to conventional MIMO radar systems.

The paper is organized as follows. In Section 2, the signal model of spatial-frequency diversity MIMO radar is briefly introduced. In Section 3, the water-filling algorithm is introduced to energy allocation problem of frequency channels firstly. Then, an iterative algorithm is proposed to optimize the transmitted waveforms and receiving filters alternatively in Section 4. The simulations results that show the advantages of spatial-frequency diversity MIMO radar are presented in Section 5 which is followed by the conclusions in Section 6.

\section{Signal Model of Spatial-Frequency Diversity MIMO Radar}

Consider the proposed spatial-frequency diversity MIMO radar system equipped with a transmit array of $M$ antennas and a receive array of $N$ antennas. Assume that the baseband waveform transmitted by each transmit antenna is $\mathbf{s}_{m}(m=$ $1,2, \ldots, M)$, with the snapshots of $L$. The total energy of waveforms is normalized. Each waveform $\mathbf{s}_{m}$ will be transmitted by $F$ different carrier frequencies at the same time (as shown in Figure 1). The energy of each carrier frequency is represented as $p_{f}(f=1, \ldots, F)$, and the total transmit energy is set as $E$. As shown in Figure 2, the received signal of each receive antenna is down-converted with $F$ different carrier frequencies, respectively, and summed together in baseband. Then the synthetic baseband data go through the receiving filters $\mathbf{h}_{n}(n=1,2, \ldots, N)$. 

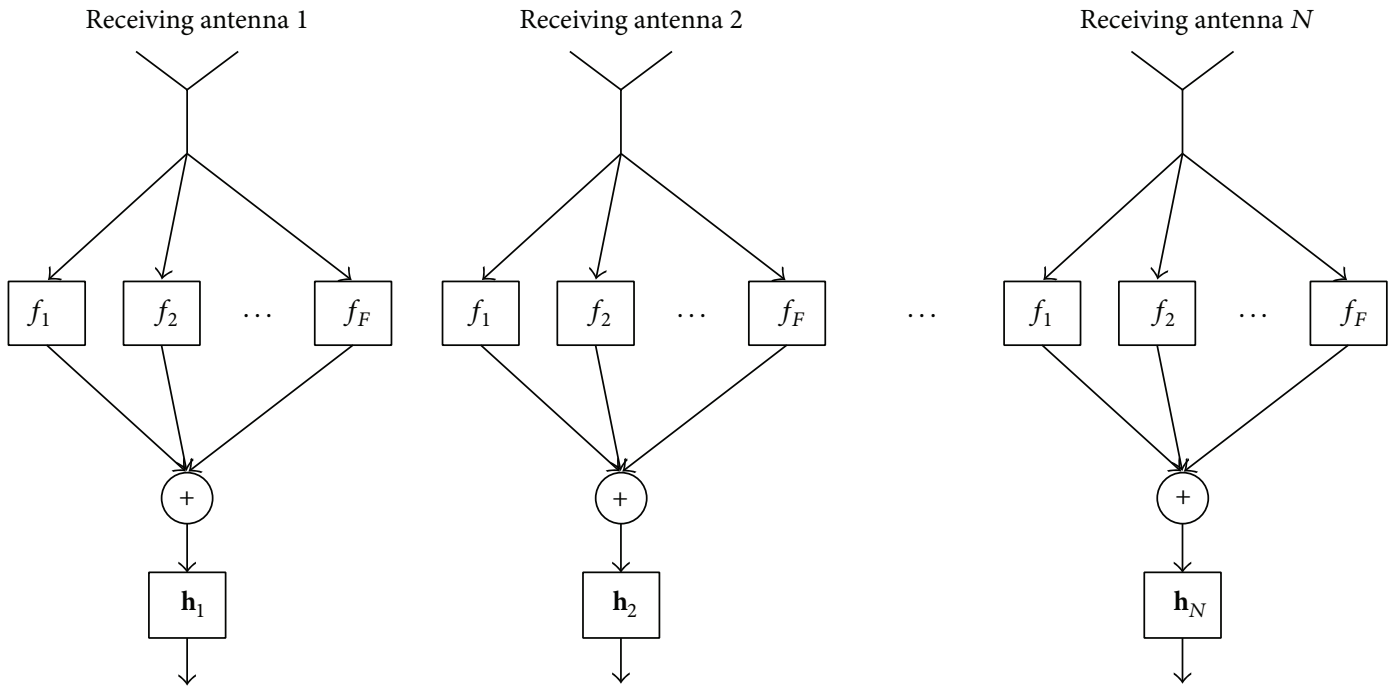

FIGURE 2: The structure of receive array.

As the proposed system satisfies (1) and (2) simultaneously, the received data of $M N F$ channels are independent. Assume that $t_{f m n}(f=1,2, \ldots, F, m=1,2, \ldots, M$, $n=1,2, \ldots, N)$ represents target reflection and attenuation coefficient between $m$ th transmit antenna and $n$th receive antenna at $f$ th frequency channel. If the target reflection and attenuation coefficients change so slowly that they can be deemed to remain constant in the $L$ snapshots duration, the received baseband target echoes at $f$ th frequency channel can be written as

$$
\mathbf{X}_{t f}=\sqrt{p_{f}} \mathbf{T}_{f} \mathbf{S}
$$

where

$$
\begin{gathered}
\mathbf{T}_{f}=\left[\begin{array}{cccc}
t_{f 11} & t_{f 12} & \cdots & t_{f 1 M} \\
t_{f 21} & t_{f 22} & \cdots & t_{f 2 M} \\
\vdots & \vdots & \ddots & \vdots \\
t_{f N 1} & t_{f N 2} & \cdots & t_{f N M}
\end{array}\right], \\
\mathbf{S}=\left[\mathbf{s}_{1}, \mathbf{s}_{2}, \ldots, \mathbf{s}_{M}\right]^{T} .
\end{gathered}
$$

The received baseband target echoes in (3) can be vectorized as

$$
\mathbf{x}_{t f}=\operatorname{vec}\left(\mathbf{X}_{t f}\right)=\sqrt{p_{f}}\left(\mathbf{I}_{L} \otimes \mathbf{T}_{f}\right) \cdot \operatorname{vec}(\mathbf{S})=\sqrt{p_{f}} \widetilde{\mathbf{T}}_{f} \mathbf{S},
$$

where $\operatorname{vec}(\cdot)$ denotes vectorized operator, $\otimes$ denotes Kronecker product, and $\mathbf{I}_{L}$ is $L \times 1$ identity matrix. Note that if target reflection and attenuation coefficients change so quickly that they will vary within the $L$ snapshots, $\widetilde{\mathbf{T}}_{f}$ should be redefined as

$$
\widetilde{\mathbf{T}}_{f}=\left[\begin{array}{cccc}
\mathbf{T}_{f 1} & \mathbf{0} & \ldots & \mathbf{0} \\
\mathbf{0} & \mathbf{T}_{f 2} & \cdots & \mathbf{0} \\
\vdots & \vdots & \ddots & \vdots \\
\mathbf{0} & \mathbf{0} & \cdots & \mathbf{T}_{f L}
\end{array}\right]_{N L \times M L}
$$

In this case, the target reflection and attenuation coefficients $\mathbf{T}_{f}$ can be assumed as stochastic processes of $M N$ random variables under the $f$ th frequency, and the $M N$ random variables may be assumed independent and identically distributed (i.i.d.), with distribution $\mathscr{C} \mathcal{N}\left(\mathbf{0}, \mathbf{R}_{\mathrm{T} f}\right)$.

Then, the sum of received baseband target echoes of all the frequency channels can be described as

$$
\mathbf{x}_{t}=\sum_{f=1}^{F} \mathbf{x}_{t f}=\sum_{f=1}^{F} \sqrt{p_{f}} \widetilde{\mathbf{T}}_{f} \mathbf{s} .
$$

Defining $\mathbf{T}=\sum_{f=1}^{F} \sqrt{p_{f}} \widetilde{\mathbf{T}}_{f},(7)$ can be rewritten as

$$
\mathbf{x}_{t}=\text { Ts. }
$$

Similarly, the clutter coefficients of $M N$ channels under $f$ th frequency also can be assumed as stochastic processes, with distribution $\left(\mathbf{0}, \mathbf{R}_{\mathbf{C} f}\right)$. By defining the clutter coefficients matrix $\widetilde{\mathbf{C}}_{f}$ which has the same structure as $\widetilde{\mathbf{T}}_{f}$ and $\mathbf{C}=$ $\sum_{f=1}^{F} \sqrt{p_{f}} \widetilde{\mathbf{C}}_{f}$, the sum of received baseband clutters of all the frequency channels can be described as

$$
\mathbf{x}_{c}=\text { Cs. }
$$

Then, the complete received baseband data can be expressed as

$$
\mathbf{x}=\mathbf{x}_{t}+\mathbf{x}_{c}+\mathbf{w}=\mathbf{T s}+\mathbf{C s}+\mathbf{w},
$$

where $\mathbf{w}$ denotes Gaussian noise with energy of $\sigma_{\mathbf{w}}^{2}$. Let the received baseband data go through the receiving filters $\mathbf{h}_{n}(n=1,2, \ldots, N)$. The output of the filters can be written as

$$
\mathbf{y}=\mathbf{h}^{T} \mathbf{x}=\mathbf{h}^{T} \mathbf{T s}+\mathbf{h}^{T} \mathbf{C s}+\widetilde{\mathbf{w}},
$$

where $\mathbf{h}=\left[\mathbf{h}_{1}^{T}, \mathbf{h}_{2}^{T}, \ldots, \mathbf{h}_{N}^{T}\right]^{T}$ with a size of $N L \times 1$.

Note that when $F$ is chosen as 1 , the signal model proposed above will be equal to the model in [18] which is based on spatial diversity MIMO radar. Consequently, the signal model of spatial diversity MIMO radar can be regarded as a special case of the proposed signal model in this paper. 


\section{Energy Allocation of Frequency Channels}

In some clutter scenarios, such as ground clutters and sea clutters, the clutter coefficients may vary with different frequencies. Especially when the frequency difference $\Delta f$ is large enough, the clutter coefficients of different frequency channels will be particularly different [20]. As a result, conventional uniform energy distribution method for frequency channels cannot achieve the best performance of spatialfrequency diversity MIMO radar.

Water-filling algorithm is widely applied in energy allocation in multichannel wireless communications, which can allot adaptively transmitted energy according to the channel condition $[21,22]$. Inspired by the theory in MIMO communication system, we introduce the water-filling algorithm to the energy allocation problem of frequency channels for spatial-frequency diversity MIMO radar. According to [22], the capacity of all the frequency channels can be described as

$$
I=\sum_{f=1}^{F} \log _{2}\left(1+\frac{p_{f} \lambda_{f}}{\sigma_{f}^{2}}\right)
$$

where $\lambda_{f} \triangleq \operatorname{trace}\left(\mathbf{R}_{\mathbf{T} f}\right), \sigma_{f}^{2} \triangleq \operatorname{trace}\left(\mathbf{R}_{\mathbf{C} f}\right)+\sigma_{\mathbf{w}}^{2}$, and $\operatorname{trace}(\cdot)$ denotes the trace of a matrix.

With the channel knowledge known, the maximum capacity of MIMO channel can be achieved by applying water-filling principle on the channel energy allocation. The optimal energy allocation $p_{f}$ is decided according to the water-filling rule [23]:

$$
p_{f}=\max \left(\mu-\frac{\sigma_{f}^{2}}{\lambda_{f}}, 0\right),
$$

where $\mu$ is a constant which guarantees the energy constraint at transmitter that

$$
\sum_{f=1}^{F} p_{f}=E
$$

\section{Joint Optimization of Transmitted Waveforms and Receiving Filters}

Spatial-frequency diversity MIMO radar provides much more extra degrees of freedom to design transmitted waveforms and receiving filters. Even in clutter environment, it is possible to improve the output SCNR of the proposed system, as long as the transmitted waveforms and receiving filters are designed properly. In this section, the rule of maximizing the output SCNR is applied to optimize the transmitted waveforms and receiving filters jointly.

Define the output SCNR of system as

$$
\operatorname{SCNR}=\frac{E\left[\left|\mathbf{h}^{T} \mathbf{T s}\right|^{2}\right]}{E\left[\left|\mathbf{h}^{T} \mathbf{C s}\right|^{2}\right]+E\left[|\widetilde{\mathbf{w}}|^{2}\right]} .
$$

As the rule of maximizing SCNR is applied, the optimization problem can be expressed as

$$
\max _{\mathbf{s}, \mathbf{h}} \frac{\mathbf{h}^{T} E\left[\mathbf{T s s}^{T} \mathbf{T}^{T}\right] \mathbf{h}}{\mathbf{h}^{T} E\left[\mathbf{C s s}^{T} \mathbf{C}^{T}\right] \mathbf{h}+E\left[|\widetilde{\mathbf{w}}|^{2}\right]} \quad \text { s.t. }\|\mathbf{s}\|^{2}=1 .
$$

Obviously, the optimization is not a convex problem and cannot get its global optimal solutions easily. However, several local optimal solutions may be found within the feasible solution space. In this paper, an iterative algorithm which optimizes the transmitted waveforms $\mathbf{s}$ and receiving filters $\mathbf{h}$ alternatively is proposed to improve the SCNR. The numerical simulation results show that each step of the proposed algorithm is nondecreasing, so that the convergence of the proposed method is guaranteed. Although the final results of the iteration algorithm may be just local optimum, they will be global optimum with respect to $\mathbf{s}$ dimension and $\mathbf{h}$ dimension, respectively. As a result, the optimized result is the best one among the multiple local optimal solutions.

Firstly, we solve $\mathbf{h}$ in terms of $\mathbf{s}$. In this case, the optimization problem above can be written as

$$
\max _{\mathbf{h}} \frac{\mathbf{h}^{T} E\left[\mathbf{T s s}^{T} \mathbf{T}^{T}\right] \mathbf{h}}{\mathbf{h}^{T} E\left[\mathbf{C s s}^{T} \mathbf{C}^{T}\right] \mathbf{h}+\mathbf{h}^{T} E\left[\mathbf{w} \mathbf{w}^{T}\right] \mathbf{h}} .
$$

Define

$$
\begin{aligned}
\mathbf{R}_{\mathrm{Ts}} \triangleq E\left[\mathrm{Tss}^{T} \mathbf{T}^{T}\right], \\
\mathbf{R}_{\mathbf{C s}} \triangleq E\left[\mathbf{C s s}^{T} \mathbf{C}^{T}\right], \\
\mathbf{R}_{\mathbf{w}} \triangleq E\left[\mathbf{w} \mathbf{w}^{T}\right] .
\end{aligned}
$$

Then, (17) can be rewritten as

$$
\max _{\mathbf{h}} \frac{\mathbf{h}^{T} \mathbf{R}_{\mathrm{Ts}} \mathbf{h}}{\mathbf{h}^{T}\left(\mathbf{R}_{\mathrm{Cs}}+\mathbf{R}_{\mathrm{w}}\right) \mathbf{h}} .
$$

It is obvious that (19) is the well-known Rayleigh quotient problem [24] and the solution to this problem is the principal generalized eigenvector of $\mathbf{R}_{\mathrm{Ts}}$ and $\left(\mathbf{R}_{\mathrm{Cs}}+\mathbf{R}_{\mathrm{w}}\right)$; that is,

$$
\widetilde{\mathbf{h}}=\lambda_{\max }\left(\mathbf{R}_{\mathrm{Ts}}, \mathbf{R}_{\mathrm{Cs}}+\mathbf{R}_{\mathrm{w}}\right),
$$

where $\lambda_{\max }(\mathbf{A}, \mathbf{B})$ denotes the generalized eigenvector corresponding to the maximum generalized eigenvalue of $\mathbf{A}$ and B.

To solve $\mathbf{s}$ in terms of $\mathbf{h}$, the optimization problem can be rewritten as

$$
\max _{\mathbf{s}} \frac{\mathbf{s}^{T} E\left[\mathbf{T}^{T} \mathbf{h} \mathbf{h}^{T} \mathbf{T}\right] \mathbf{s}}{\mathbf{s}^{T} E\left[\mathbf{C}^{T} \mathbf{h} \mathbf{h}^{T} \mathbf{C}\right] \mathbf{s}+\mathbf{h}^{T} E\left[\mathbf{w} \mathbf{w}^{T}\right] \mathbf{h}} \quad \text { s.t. }\|\mathbf{s}\|^{2}=1 .
$$

Similarly, define

$$
\begin{aligned}
& \mathbf{R}_{\mathbf{T h}} \triangleq E\left[\mathbf{T}^{T} \mathbf{h} \mathbf{h}^{T} \mathbf{T}\right], \\
& \mathbf{R}_{\mathbf{C h}} \triangleq E\left[\mathbf{C}^{T} \mathbf{h} \mathbf{h}^{T} \mathbf{C}\right] .
\end{aligned}
$$


Then, (21) can be rewritten as

$$
\max _{\mathbf{s}} \frac{\mathbf{s}^{T} \mathbf{R}_{\mathbf{T h}} \mathbf{s}}{\mathbf{s}^{T} \mathbf{R}_{\mathbf{C h}} \mathbf{s}+\mathbf{h}^{T} \mathbf{R}_{\mathbf{w}} \mathbf{h}} \quad \text { s.t. }\|\mathbf{s}\|^{2}=1 .
$$

Unfortunately, the structure of (23) is slightly different from (19), and it is not a standard Rayleigh quotient problem. However, noting the fact that the constraint satisfies $\|\mathbf{s}\|^{2}=$ $\mathbf{s}^{T} \mathbf{s}=1$, the optimization problem (23) is equal to

$$
\max _{\mathbf{s}} \frac{\mathbf{s}^{T} \mathbf{R}_{\mathbf{T h}} \mathbf{s}}{\mathbf{s}^{T} \mathbf{R}_{\mathbf{C h}} \mathbf{s}+\mathbf{s}^{T}\left(\mathbf{h}^{T} \mathbf{R}_{\mathbf{w}} \mathbf{h} \cdot \mathbf{I}_{M L}\right) \mathbf{s}},
$$

where $\mathbf{I}_{M L}$ denotes identity matrix with a size of $M L$. Equation (24) is a Rayleigh quotient problem and its solution satisfies the constraint $\|\mathbf{s}\|^{2}=1$. As a result, the optimal solution to (23) can be computed by

$$
\widetilde{\mathbf{s}}=\lambda_{\max }\left(\mathbf{R}_{\mathbf{T h}}, \mathbf{R}_{\mathbf{C h}}+\mathbf{h}^{T} \mathbf{R}_{\mathbf{w}} \mathbf{h} \cdot \mathbf{I}_{M L}\right) .
$$

All steps of the proposed algorithm are summarized as follows.

(1) Compute transmitted energy allocation $p_{f}$ of different frequency channels by (13).

(2) Initialize the transmitted waveforms s (e.g., random waveforms).

(3) Compute $\mathbf{R}_{\mathrm{Ts}}, \mathbf{R}_{\mathrm{Cs}}$, and $\mathbf{R}_{\mathbf{w}}$ by (18).

(4) Compute $\mathbf{h}$ by (20).

(5) Compute $\mathbf{R}_{\mathbf{T h}}$ and $\mathbf{R}_{\mathbf{C h}}$ by (22).

(6) Compute $s$ by (25).

(7) Compute the SCNR by (15), and compute the difference $\sigma$ between the two adjacent iterative results of SCNR.

(8) Repeat steps 3-7 until the difference $\sigma$ is less than a very small value (e.g., $10^{-4}$ ).

\section{Simulation Results}

In this section, some simulations are performed to show the performance of spatial-frequency diversity MIMO radar against clutters. Throughout our experiments, a MIMO radar system with a transmit array of $M=4$ and a receive array of $N=4$ is assumed. The configurations of arrays satisfy (1) and (2). The number of snapshots is set as $L=16$ and the energy of Gaussian noise is assumed as $\sigma_{\mathrm{w}}^{2}=1$. In the experiments of SCNR versus CNR (clutter-to-noise ratio), each method has completed 200 independent runs.

Experiment 1. In this experiment, the number of carrier frequencies are assumed as $F=3$. The transmitted waveforms are initialized as random waveforms. The total transmitted energy of all frequency channels are set as $E=1$. The coefficients $\left\{t_{f m n}\right\}$ are generated as independent and identically distributed Gaussian random variables with unity variance;

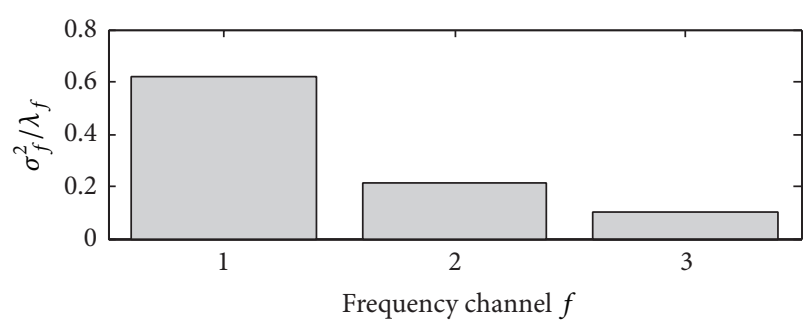

(a)

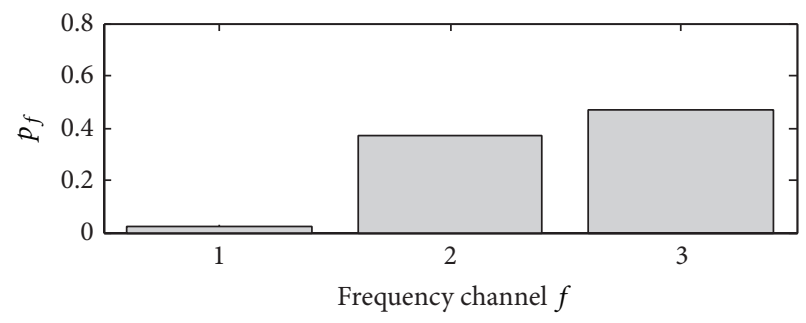

(b)

FIGURE 3: The conditions and transmitted energy allocation of frequency channels.

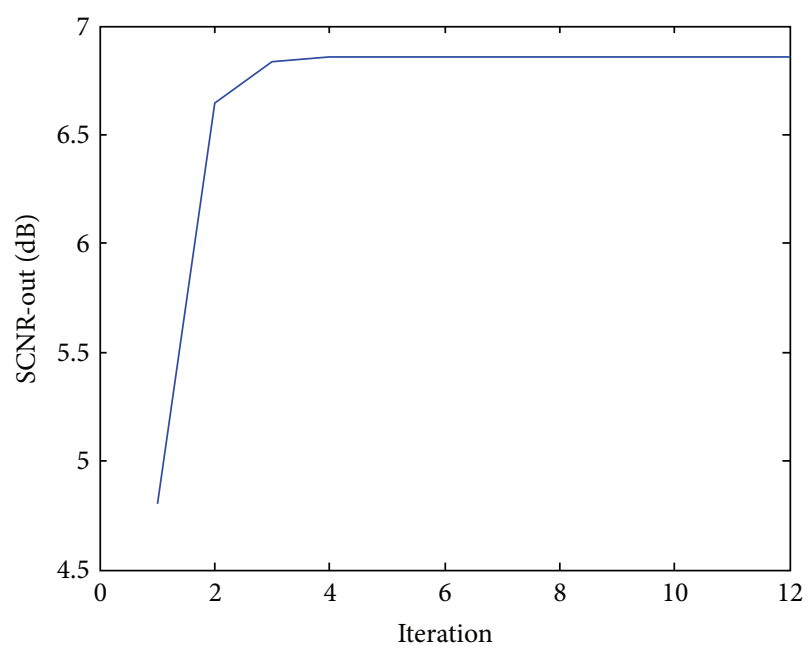

FIGURE 4: The output SCNR versus the number of iterations.

namely, $\mathbf{R}_{\mathbf{T} f}=\mathbf{I}_{M N}$. The covariance matrixes $\mathbf{R}_{\mathbf{C} f}$ of clutters under each frequency channel are generated by

$$
\mathbf{R}_{\mathbf{C}_{f}}=\mathbf{B A}^{f} \mathbf{B}, \quad f=1,2,3,
$$

where $\mathbf{A}$ is a positive semidefinite matrix with a size of $16 \times 16$ whose maximum spectral radius is no more than 1 and $\mathbf{B}$ is a unitary matrix with a size of $16 \times 16$. Note that $(26)$ with superscript $f$ applied here is just for the aim of generating the different clutter covariance matrixes in different frequency channels.

By applying the water-filling algorithm, the energy allocation of each frequency channel is shown in Figure 3. According to the condition of each frequency channel, the energy is no longer uniformly distributed. Then, the proposed iterative algorithm (from (18) to (25)) is applied to optimize the system output SCNR. Figure 4 shows the trends of SCNR 


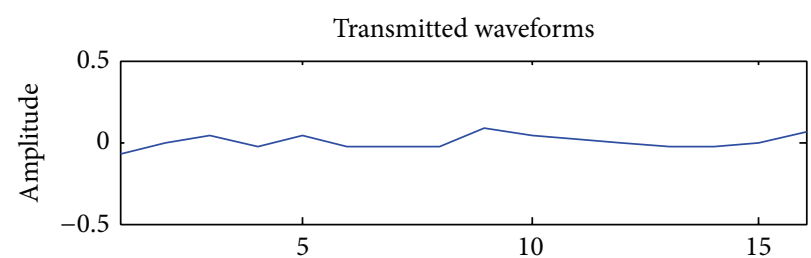

(a)

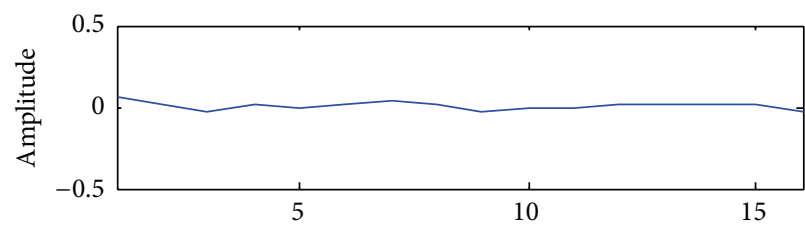

(b)

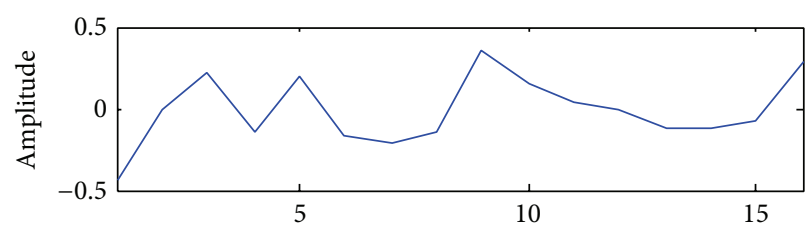

(c)

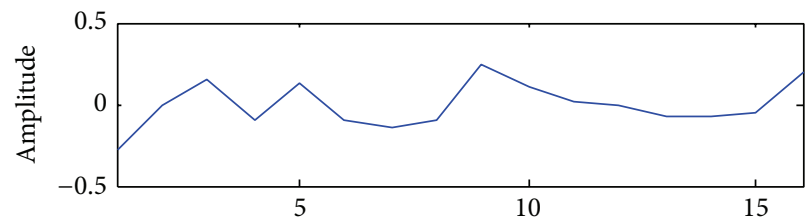

(d)

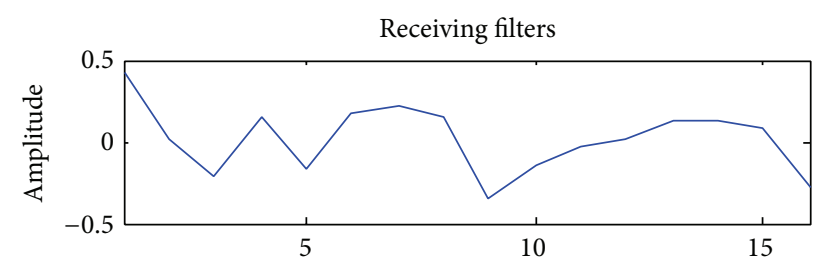

(e)

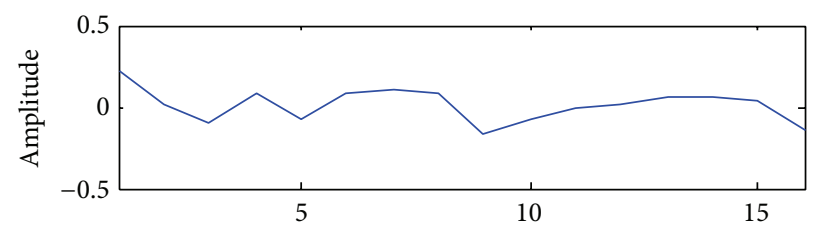

(f)

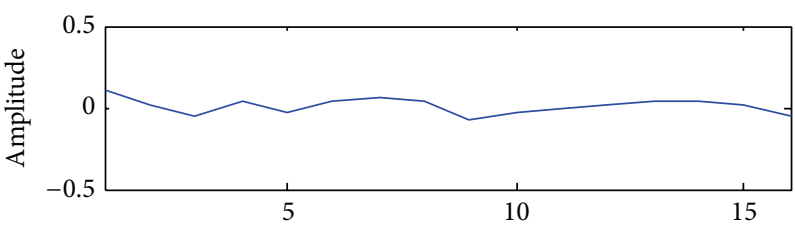

(g)

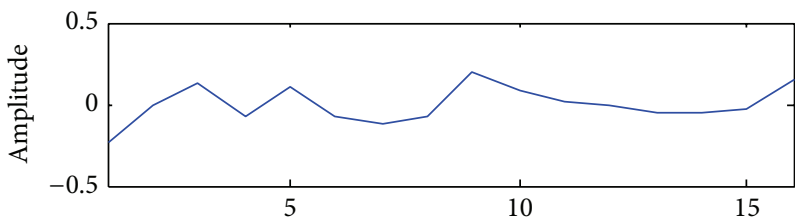

(h)

FIGURE 5: The optimized results: (a)-(d) are transmitted waveforms and (e)-(f) are receiving filters.

versus iterative number. It can be found that the SCNR in each step of the proposed iterative algorithm is nondecreasing and rapid convergence of the method is guaranteed. The optimized transmitted waveforms and receiving filters are shown in Figure 5.

Experiment 2. In order to illustrate the effects of water-filling algorithm and the number $F$ of frequency channels on the performance of the system, we also perform the simulations of the output SCNR versus input CNR in five cases: (i) $F=$ 2 with applying the water-filling algorithm; (ii) $F=3$ with applying the water-filling algorithm; (iii) $F=2$ with applying uniform energy allocation method; (iv) $F=3$ with applying uniform energy allocation method; (v) the method for spatial diversity MIMO radar in [18] $(F=1)$.

For simplicity, the target coefficients are assumed as

$$
t_{\text {fnm }}=1, \quad n, m=1, \ldots, 4, f=1,2,3 .
$$

The clutter covariance matrixes $\mathbf{R}_{\mathbf{C} f}$ are generated similarly to that in Experiment 1. The total transmitted energy of all frequency channels are set as $E=1$. However, the input CNR varies in the range $[-5 \mathrm{~dB}, 30 \mathrm{~dB}]$. The simulation results of the output SCNR versus input CNR in the cases of $F=2$ and $F=3$ with applying water-filling algorithm or uniform energy allocation method are shown in Figure 6. Obviously, the two cases which apply the water-filling algorithm have better performance than the other two with uniform energy allocation. It is demonstrated that the water-filling algorithm works very well in spatial-frequency diversity MIMO radar. Besides, the performance in the case of $F=3$ is also much better than that in the cases of $F=1$ and 2, which shows that more frequency diversities will make more contributions to the system performance. Nevertheless, we could not increase the number of carrier frequencies without restrictions. The reason is that the increased number of carrier frequencies will not only result in extra hardware cost and increased usage of electromagnetic spectrum but also lead to the increase of computational complexity. Consequently, it is still a problem that is worthy of further study to seek the balance among the number of carrier frequencies, hardware cost, computational complexity, and the increase of system performance.

Experiment 3. In $[3,4]$, the authors also proposed a method to design optimal waveforms by only exploiting spatial diversity. In their proposed algorithm, water-filling algorithm is used to optimize the transmitted power from different antennas, whereas similar algorithm is used to optimize the transmitted power form different frequency channels in our method. Besides, their method can also achieve the closedform optimal solution; namely, the left singular vectors of the optimal waveform should be the eigenvectors of the covariance matrix of colored noise and the right singular vectors should be eigenvectors of the covariance matrix 


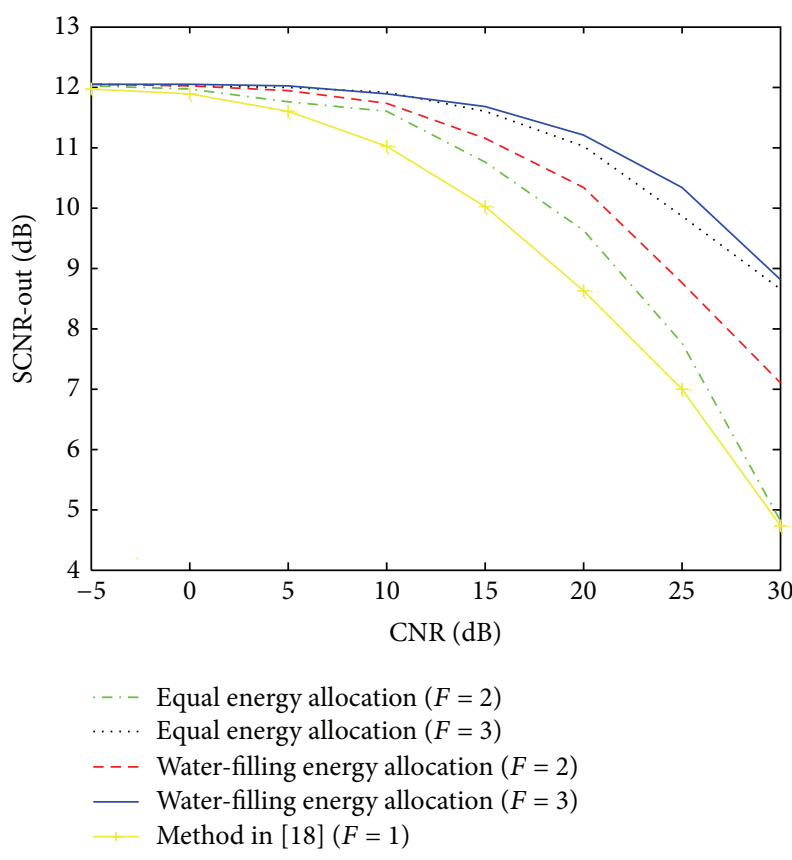

Figure 6: The output SCNR versus input CNR under five cases.

of target. To illustrate the superiority of our method, the comparison in signal-dependent scenario of the two methods under the rule of maximizing SCNR is performed. Note that the application scenario discussed in this paper is signaldependent clutter suppression, so that the comparison of signal-independent noise suppression performance for the two methods is not considered here.

In this experiment, the MIMO radar equipped with $M=$ 4 widely distributed arrays is assumed. The eigenvalues of covariance matrix of target $\mathbf{R}_{H}$ (which is defined in [4]) are set as $\{7,5,2,1\}$. The clutter covariance matrixes $\mathbf{R}_{\mathbf{C} f}$ and noise are generated similarly to that in Experiment 2 . The input CNR varies in the range $[-5 \mathrm{~dB}, 30 \mathrm{~dB}]$. The total transmit energy is set as $E=100$. Note that the signaldependent clutter and noise here are treated as the colored noise part $\mathbf{W}$ (which is defined in [4]) in the simulation of the method in [4]. In our method, the number of carrier frequencies is set as $F=3$. The trends of SCNR versus CNR of the two methods are shown in Figure 7. The result of the method applying orthogonal waveforms and matched filters is also drawn in Figure 7. It is obvious that the performance of our proposed method is much better than that in [4], and the performance of the method in [4] is similar to the matched filters method with no anticlutter measures applied. It demonstrates that the method in [4] is unable to suppress the signal-dependent clutter effectively, although it works well in the colored noise suppression application (where the noise part is independent of transmit signal). In signal-dependent clutter suppression application, the performance of the method we proposed is much better than conventional MIMO radar and those in [4]. However, the computational complexity of our method will increase linearly with the number of iterations. Compared with the

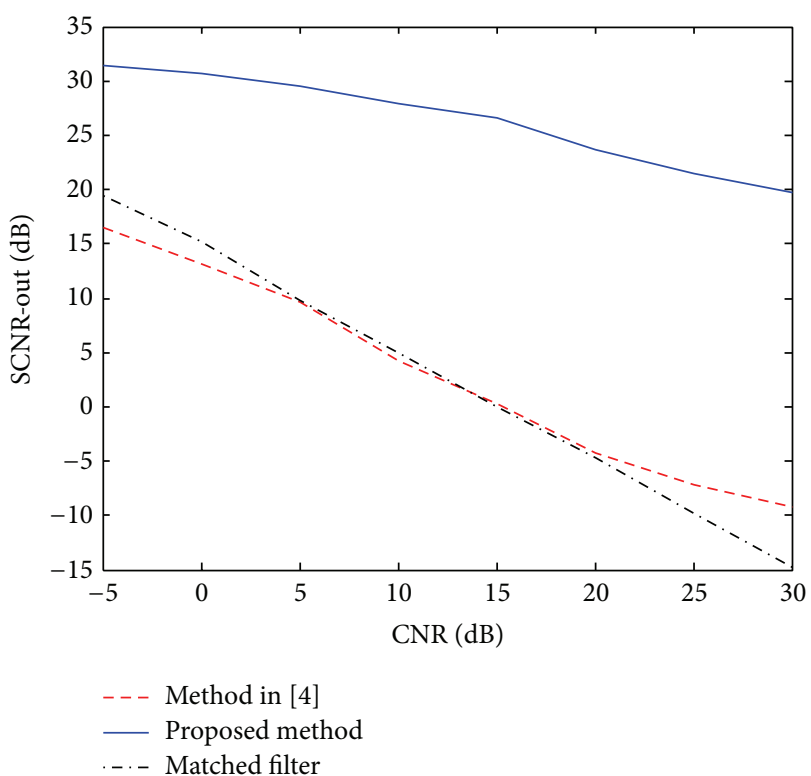

FIgURE 7: The comparison of the SCNR versus CNR.

closed-form solution in [4], more computing resources are needed in the proposed method.

Experiment 4. In this experiment, two application scenes for the proposed method are investigated: the target reflection and attenuation coefficients slowly changed case and quickly changed case. For the slowly changed target case, the coefficients $\left\{t_{f m n}\right\}$ are deemed to remain constant in $L$ snapshots duration, so that the FMN coefficients are assumed as independent and identically distributed Gaussian random variables with unity variance. For the quickly changed target case, the coefficients $\left\{t_{f m n}\right\}$ will vary within $L$ snapshots. Similarly to Experiment 1 , the LFMN coefficients in this case are generated as independent and identically distributed Gaussian random variables with unity variance too. For both cases, the $F$ is set as 3 . Under the same MIMO radar system and clutter assumption in Experiment 2, the simulation results of the output SCNR versus input CNR in both cases are shown in Figure 8. It shows that our method has a better performance in quickly changed target case, for the reason that more RCS samples of quickly changed target are obtained in time domain. It is demonstrated once again that MIMO radar has the ability to suppress the target scintillation effectively and the advantages of diversity information brought by MIMO radar can effectively improve the target detection performance.

\section{Conclusions}

In this paper, the spatial and frequency diversities are combined in one MIMO radar system, namely, spatial-frequency diversity MIMO radar. By taking full advantages of the extra degrees of freedom brought by plenty of spatial and frequency diversities, we investigate the application of spatial-frequency 


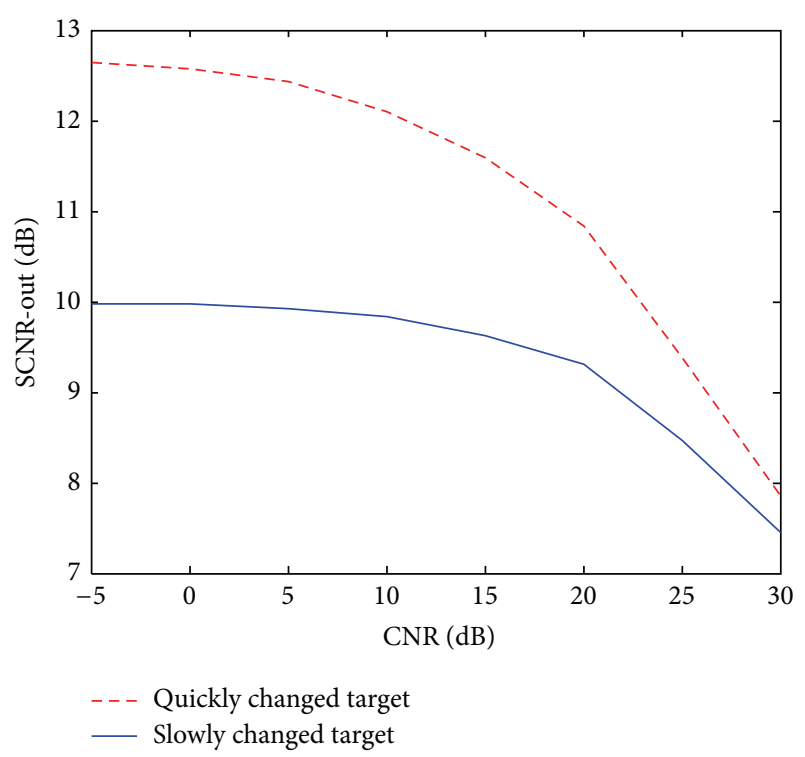

FIGURE 8: The output SCNR versus input CNR in slowly and quickly changed target cases.

diversity MIMO radar in clutter scenarios. Firstly, the waterfilling algorithm is introduced to the energy allocation problem of frequency channels. The simulation result shows that it works well to improve the system performance. Then, an iterative algorithm which optimizes the transmitted waveforms and receiving filters alternatively is proposed to maximize the output SCNR, which will improve the target detection performance in the presence of clutters. Simulation results show that the methods we proposed can effectively suppress the clutters with spatial-frequency diversity MIMO radar. Compared with existing methods, the proposed method has a superior performance in the presence of clutter. In addition, it is demonstrated once again in our simulation results that MIMO radar can suppress the target scintillation effectively.

\section{Conflict of Interests}

The authors declare that there is no conflict of interests regarding the publication of this paper.

\section{Acknowledgments}

This work is supported in part by the National Natural Science Foundation of China (Grant no. 11273017), the College Graduate Scientific Research Innovation Fund in Jiangsu Province of China (Grant no. CXZZ13_0205), and the Shanghai Aerospace Science and Technology Innovation Fund of China (Grant no. SAST201356).

\section{References}

[1] W. Zhang and L. Yang, "Communications-inspired sensing: a case study waveform design," IEEE Transactions on Signal Processing, vol. 58, no. 2, pp. 792-803, 2010.
[2] Y. Yang and R. S. Blum, "MIMO radar waveform design based on mutual information and minimum mean-square error estimation," IEEE Transactions on Aerospace and Electronic Systems, vol. 43, no. 1, pp. 330-343, 2007.

[3] B. Tang, J. Tang, and Y. Peng, "MIMO radar waveform design in colored noise based on information theory," IEEE Transactions on Signal Processing, vol. 58, no. 9, pp. 4684-4697, 2010.

[4] B. Tang, J. Tang, and Y. Peng, "Waveform optimization for MIMO radar in colored noise: further results for estimationoriented criteria," IEEE Transactions on Signal Processing, vol. 60, no. 3, pp. 1517-1522, 2012.

[5] Y. C. Wang, X. Wang, H. Liu, and Z. Luo, "On the design of constant modulus probing signals for MIMO radar," IEEE Transactions on Signal Processing, vol. 60, no. 8, pp. 4432-4438, 2012.

[6] F. Daum and J. Huang, "MIMO radar: snake oil or good idea?" in Proceedings of the IEEE International Waveform Diversity and Design Conference (WDD '09), pp. 113-117, Kissimmee, Fla, USA, February 2009.

[7] E. Fishler, A. Haimovich, R. Blum, D. Chizhik, L. Cimini, and R. Valenzuela, "MIMO radar: an idea whose time has come," in Proceedings of the IEEE Radar Conference, pp. 71-78, April 2004.

[8] C. Du, J. S. Thompson, and Y. R. Petillot, "Detector and waveform design for MIMO radar system with noisy channel estimation," IEEE Transactions on Aerospace and Electronic Systems, vol. 48, no. 3, pp. 2332-2348, 2012.

[9] T. Naghibi and F. Behnia, "MIMO radar waveform design in the presence of clutter," IEEE Transactions on Aerospace and Electronic Systems, vol. 47, no. 2, pp. 770-781, 2011.

[10] Y. Yang and R. S. Blum, "Minimax robust MIMO radar waveform design," IEEE Journal on Selected Topics in Signal Processing, vol. 1, no. 1, pp. 147-155, 2007.

[11] E. Grossi, M. Lops, and L. Venturino, "Robust waveform design for MIMO radars," IEEE Transactions on Signal Processing, vol. 59, no. 7, pp. 3262-3271, 2011.

[12] A. M. Haimovich, R. S. Blum, and L. J. Cimini, "MIMO radar with widely separated antennas," IEEE Signal Processing Magazine, vol. 25, no. 1, pp. 116-129, 2008.

[13] E. Fishler, A. Haimovich, R. S. Blum, L. J. Cimini Jr., D. Chizhik, and R. A. Valenzuela, "Spatial diversity in radars-models and detection performance," IEEE Transactions on Signal Processing, vol. 54, no. 3, pp. 823-838, 2006.

[14] V. Ravenni, "Performance evaluations of frequency diversity radar system," in Proceedings of the 37th IEEE European Microwave Conference (EUMC'07), pp. 1715-1718, October 2007.

[15] J. J. Zhang and A. Papandreou-Suppappola, "MIMO radar with frequency diversity," in Proceedings of the International Waveform Diversity and Design Conference (WDD '09), pp. 208212, IEEE, Kissimmee, Fla, USA, February 2009.

[16] S. H. Zhou and H. W. Liu, "Target statistical correlation characteristic for spatial-frequency jointly diversity multipleinput multiple-output radar," IET Radar, Sonar and Navigation, vol. 5, no. 6, pp. 638-649, 2011.

[17] E. F. Nathanson, P. J. Reilly, and M. N. Cohen, "Radar design principles-Signal processing and the Environment," NASA STI/Recon Technical Report A, vol. 91, article 46747, 1991.

[18] B. Friedlander, "Waveform design for MIMO radars," IEEE Transactions on Aerospace and Electronic Systems, vol. 43, no. 3, pp. 1227-1238, 2007. 
[19] A. Aubry, A. Demaio, A. Farina, and M. Wicks, "Knowledgeaided (potentially cognitive) transmit signal and receive filter design in signal-dependent clutter," IEEE Transactions on Aerospace and Electronic Systems, vol. 49, no. 1, pp. 93-117, 2013.

[20] M. I. Skolnik, Radar Handbook, 1970.

[21] X. Ling, B. Wu, H. Wen, L. Pan, and F. Luo, "Fast and efficient parallel-shift water-filling algorithm for power allocation in orthogonal frequency division multiplexing-based underlay cognitive radios," IET Communications, vol. 7, no. 12, pp. 12691278, 2013.

[22] A. Yoshimoto and T. Hattori, "Area coverage of a multi-link MIMO system with water filling power allocation strategy," in Proceedings of the 66th IEEE Vehicular Technology Conference (VTC '07), pp. 1137-1141, IEEE, October 2007.

[23] T. M. Cover and J. A. Thomas, Elements of Information Theory, John Wiley \& Sons, 2012.

[24] R. A. Horn and C. R. Johnson, Matrix Analysis, Cambridge University Press, 2012. 

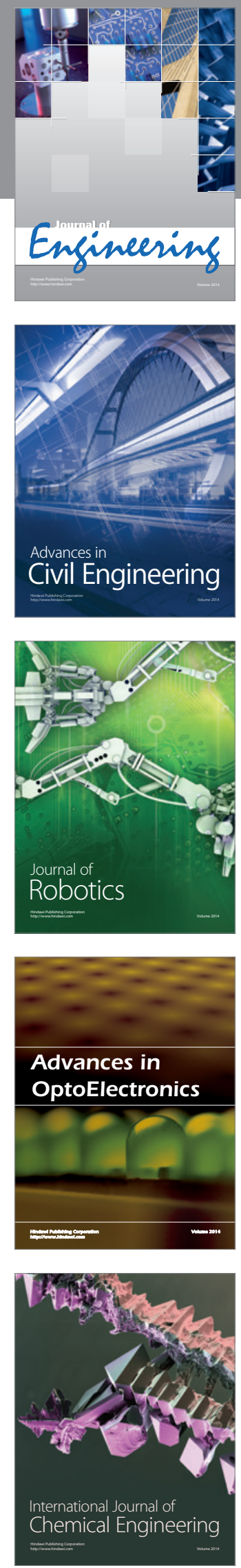

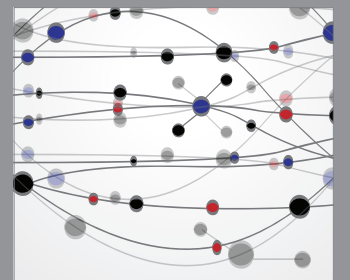

The Scientific World Journal
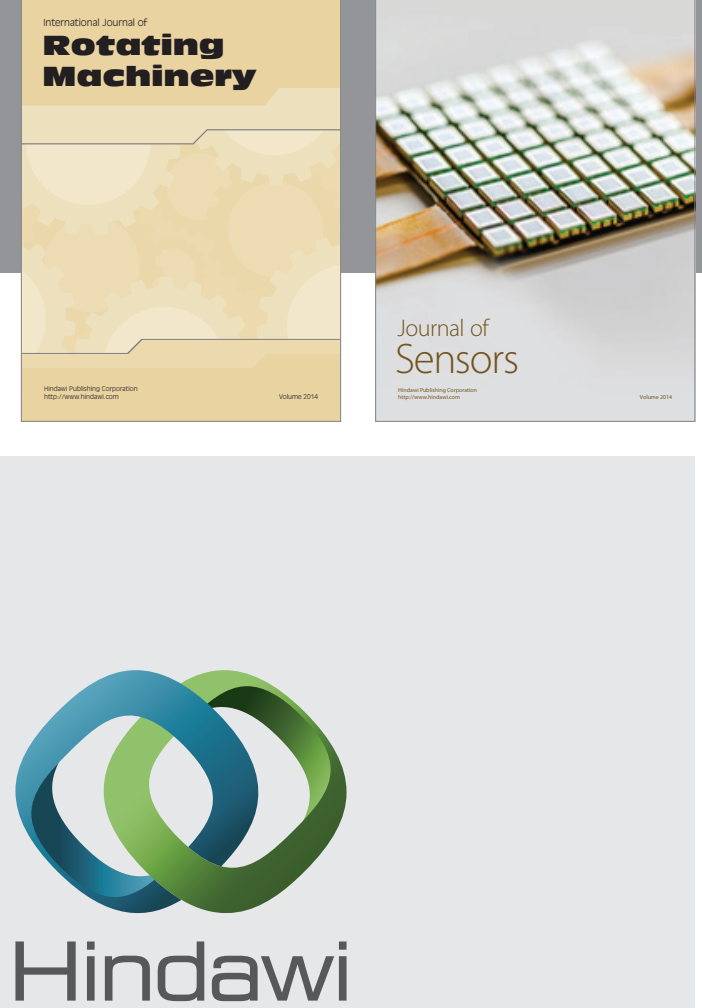

Submit your manuscripts at http://www.hindawi.com
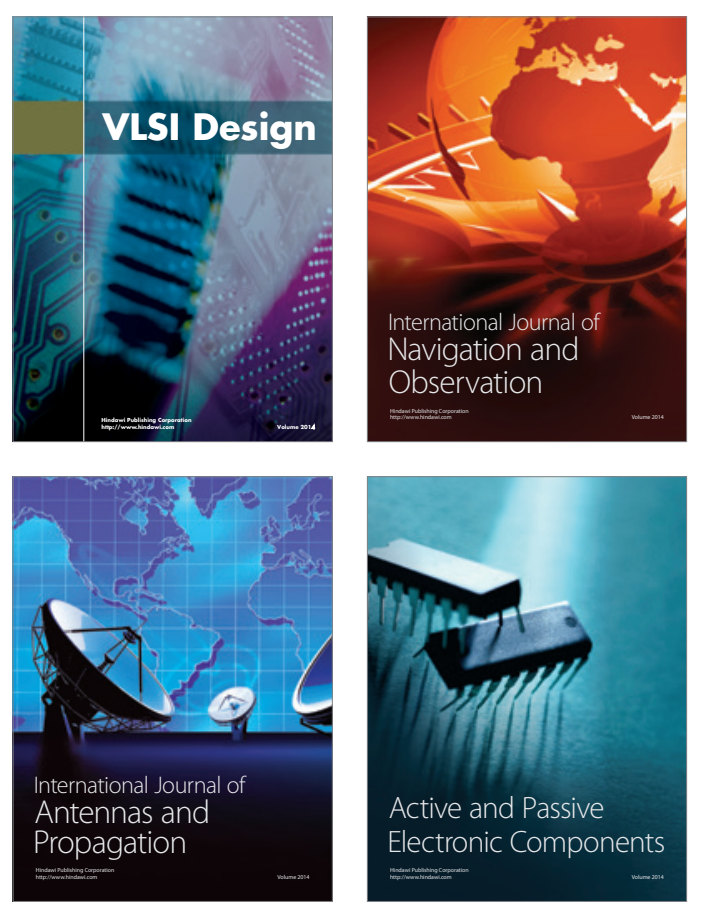
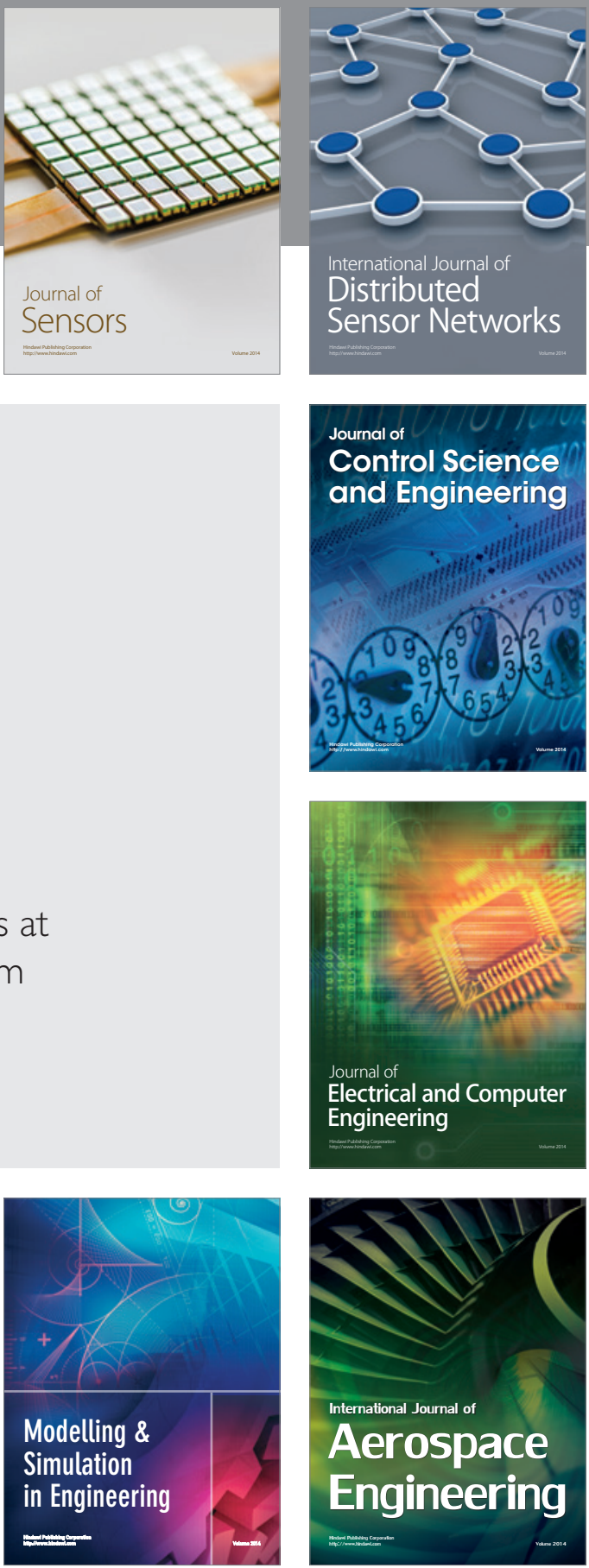

Journal of

Control Science

and Engineering
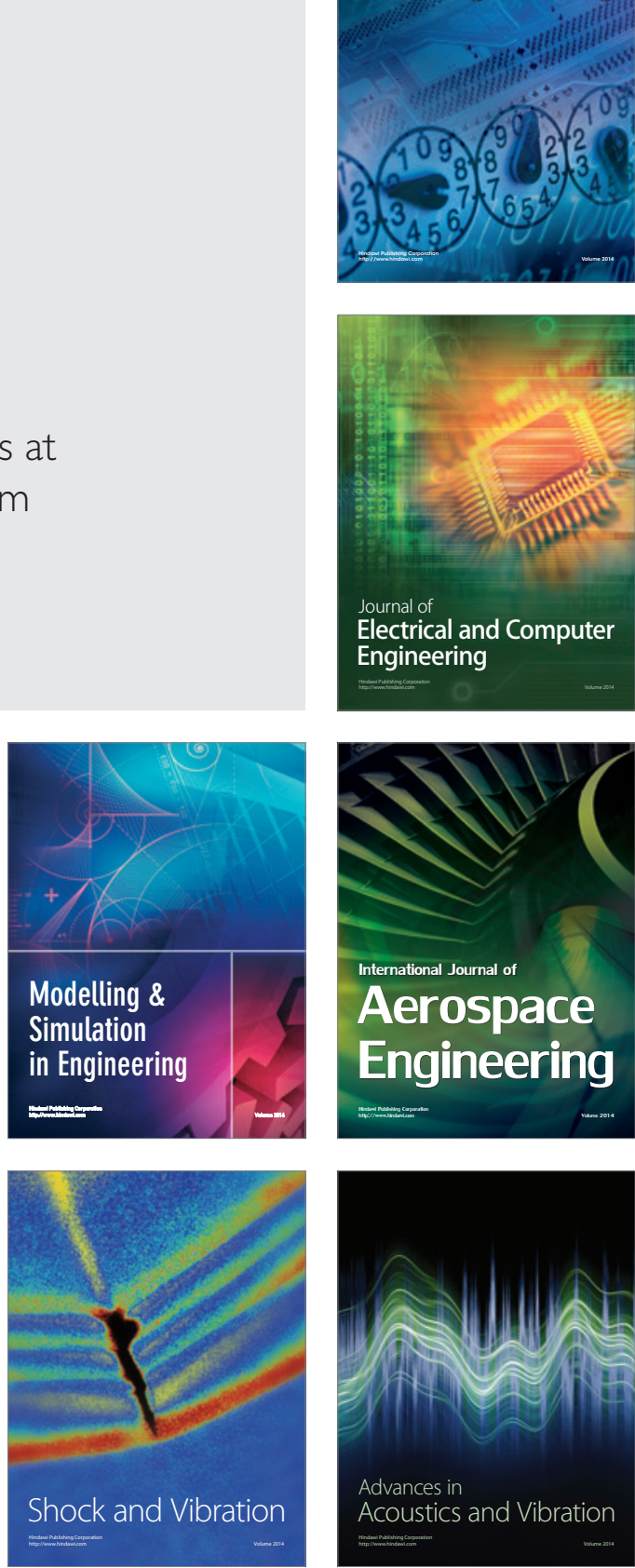